\title{
Perfil epidemiológico de la candidiasis invasora en unidades de pacientes críticos en un hospital universitario
}

\author{
M. Cristina Ajenjo H., Andrés Aquevedo S., Ana María Guzmán D., Helena Poggi M., Mario Calvo A., \\ Claudia Castillo V., Eugenia León C., Max Andresen H. y Jaime Labarca L.
}

\begin{abstract}
Pontificia Universidad Católica de Chile, Santiago. Facultad de Medicina Departamento de Medicina Interna (MCAH, JLL). Alumno de la Escuela de Medicina (AAS).

Departamento de Laboratorios Clínicos (AMGD, HPM, CCV, ELC).

Departamento de Medicina Intensiva (MAH).

Universidad Austral de Chile, Valdivia.

Instituto de Medicina (MCA).

Potenciales conflictos de interés: Este estudio fue realizado gracias a una donación del laboratorio Pfizer

Chile S.A. MCA y J han servido

como expositores y consultores

para Merck Sharp \& Dohme Chile. AA, AMG, HP, MC, CC, EL y MA no reportan potenciales conflictos de interés referentes a este estudio.
\end{abstract}

Recibido: 6 de enero de 2010 Aceptado: 29 de diciembre de 2010

Correspondencia a: M. Cristina Ajenjo Henríquez majenjo@puc.cl

\section{Introducción}

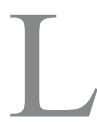
a candidiasis invasora $(\mathrm{CI})$ es un importante problema epidemiológico y clínico en las unidades de cuidados intensivos (UCI), siendo en los Estados Unidos de América (E.U.A.) la tercera causa de infecciones del torrente sanguíneo en estas unidades ${ }^{1-3}$. Esta situación es el resultado de la combinación de diversos factores, entre los cuales destaca la atención de pacientes cada vez más complejos que reúnen factores de riesgo para $\mathrm{CI}^{4-6}$, así como por la mejoría de la sobrevida de estos pacientes gracias a los avances en tratamiento intensivo.

El diagnóstico clínico de candidiasis invasora es difícil y frecuentemente tardío. Además, los criterios empleados para el diagnóstico no están claramente estandarizados. Estudios internacionales han mostrado que es posible hacer diagnóstico de CI por hemocultivos (candidemia) en sólo 20 a 50\% de los pacientes con infección demostrada en órganos profundos o por necropsia ${ }^{7,8}$. Debido a la baja sensibilidad de los hemocultivos y a la falta de exámenes de diagnóstico confiables, la mayor parte de las veces el diagnóstico es presuntivo, basado en parámetros clínicos. En estos casos, el tratamiento que es de alto costo y significativa toxicidad, es habitualmente empírico. A pesar de la introducción de nuevas terapias antifúngicas, la mortalidad atribuible a esta patología sigue siendo elevada, estimándose en cifras variables que van desde 29 a $50 \%{ }^{9}$.

La epidemiología de las infecciones por C. albicans ha ido cambiando en el mundo. En la última década se ha observado progresivamente una mayor proporción de candidemias causadas por especies de Candida no albicans $^{10-12}$, probablemente como consecuencia del aumento del consumo de fluconazol en $\mathrm{UCI}^{13}$. Este problema es particularmente importante en la elección de la terapia antifúngica a utilizar en pacientes críticos que cursen con una CI, dado que algunas especies de Candida no albicans pueden tener susceptibilidad disminuida o resistencia intrínseca a los azoles, que son los antifúngicos más utilizados ${ }^{14,15}$. Además, el retraso en el inicio de un tratamiento antifúngico empírico adecuado se ha asociado a un aumento de la mortalidad hospitalaria en pacientes con candidemia ${ }^{16,17}$.

En nuestro país, este problema se agrava aún más por la falta de información del comportamiento epidemiológico local de la CI en UCI. El objetivo de este estudio es describir el perfil epidemiológico de la CI en UCI de adultos, determinando la distribución de especies y la susceptibilidad a antifúngicos de las cepas de Candida sp encontradas en candidemias, con el fin de tener información que permita guiar la terapia antifúngica en los pacientes críticos. 
Este estudio fue realizado mediante un proyecto financiado durante un período de dos años (2001-2003) y presentado como comunicación oral en el XX Congreso Chileno de Infectología, La Serena, noviembre de 2003. Dada la escasa información científica nacional respecto al tema y revisada la literatura internacional de los últimos años, los resultados reportados están totalmente vigentes y pudieran ser de gran utilidad para guiar el tratamiento empírico de pacientes con esta patología de elevada mortalidad, pese a los recientes avances en terapia antifúngica.

\section{Pacientes y Métodos}

Estudio observacional, prospectivo, que incluyó a pacientes con 18 años de edad o más con CI, hospitalizados en UCIs e Intermedios del Hospital Clínico de la Pontificia Universidad Católica de Chile, evaluados entre octubre de 2001 y agosto de 2003. Este centro es un hospital universitario, complejo, con 400 camas aproximadamente, de las cuales alrededor de $17 \%$ eran de pacientes críticos adultos al momento del estudio.

Para el ingreso al protocolo, los pacientes debían tener al menos uno de los siguientes factores de riesgo de CI: diabetes mellitus, insuficiencia renal (creatinina $>2,0 \mathrm{mg}$ / dl), uso de antimicrobianos de amplio espectro por al menos 72 horas, uso previo de corticosteroides, uso de catéter venoso central, ventilación mecánica, hemodiálisis, nutrición parenteral total central o cirugía abdominal reciente. Se consideraron tres categorías clínicas de candidiasis invasora: Candidemia, definida como la presencia de al menos un hemocultivo positivo a levaduras; Candidiasis invasora diseminada, definida como el cuadro clínico caracterizado por un síndrome febril sin foco (temperatura axilar $\geq 37,5^{\circ} \mathrm{C}$ ) y $\sin$ respuesta a antimicrobianos de amplio espectro, en un paciente con al menos un factor de riesgo de los ya mencionados y aislamiento de levaduras en al menos dos sitios, excluyendo cavidad oral y deposiciones; Candidiasis invasora local definida por la presencia de cultivo de sitio estéril positivo a levaduras. Los criterios de exclusión fueron el uso de antifúngicos sistémicos para profilaxis o tratamiento durante la hospitalización o las 72 horas previas al ingreso al estudio, paciente portador de virus de inmunodeficiencia humana y paciente cursando neutropenia febril.

Luego de firmar un consentimiento informado, se registró la información epidemiológica, clínica y de laboratorio de cada paciente, conservando a $-70^{\circ} \mathrm{C}$ las cepas de Candida sp aisladas. La identificación de levaduras a nivel de especie se realizó mediante tubo germinativo y crecimiento a $45^{\circ} \mathrm{C}$ para C. albicans y galerías de identificación $32 \mathrm{C}$ (Biomerieux ${ }^{\circledR}$ ) para todas aquellas cepas de Candida no albicans. Además se realizó estudio de susceptibilidad, por método de difusión-dilución en agar, para fluconazol, itraconazol y anfotericina $\mathrm{B}$ utilizando Etest $^{\mathbb{B}}$ (AB Biodisk), de las cepas aisladas en candidemias.

Los datos recolectados se ingresaron y analizaron utilizando Microsoft Excel 2003. Se utilizó test de chi cuadrado para comparar variables categóricas. El estudio fue evaluado y aprobado por el Comité de Ética de la Pontificia Universidad Católica de Chile.

\section{Resultados}

De un total de 231 pacientes evaluados, 53 cumplieron criterios de ingreso al estudio. De ellos, $31(58,4 \%)$ fueron mujeres. La mediana de edad fue 67 años (rango17-92 años) y el índice de gravedad expresado en APACHE II ${ }^{18}$ alcanzó una mediana de 13 puntos (rango 0-28 puntos). Los principales factores de riesgo para CI identificados al ingreso al estudio fueron: uso de antimicrobianos previos $(100 \%)$, uso de catéter venoso central $(77,3 \%)$, nutrición parenteral central (37\%), cirugía abdominal (28\%) y corticoterapia $(20 \%)$. La mediana de estadía hospitalaria previo al diagnóstico de CI fue de 11 días (rango 0-36 días). Del total de pacientes, $18(33,9 \%)$ tuvieron candidemia, 22 $(41,5 \%)$ CI diseminada y 13 (24,5\%) CI local (Tabla 1$)$.

Del total de candidemias, identificamos ocho $C$. albicans $(44,4 \%)$ y 10 cepas de Candida no albicans $(55,6 \%)$, siendo la especie predominante $C$. tropicalis (27,7\%), seguida de C. glabrata $(11,1 \%)$ y C. krusei $(5,5 \%)$. Se documentó resistencia a fluconazol en $11,2 \%$ de las cepas aisladas en candidemias, correspondiendo a dos cepas de Candida no albicans: una C. glabrata y una C. krusei (Tabla 2).

De los 22 pacientes con CI diseminada, 15 (68,2\%) presentaron la misma especie en los sitios de colonización, siendo en catorce de ellos $C$. albicans y en uno $C$. tropicalis. De los pacientes con C. albicans, 11 tuvieron colonización en secreciones respiratorias y en orina de manera simultánea, siendo ambos sitios los más frecuentes en todo el grupo de pacientes. En $7(31,8 \%)$ pacientes se aislaron cepas discordantes en ambos sitios, siendo lo más frecuente la recuperación de $C$. albicans en secreciones respiratorias y $C$. glabrata en orina, de manera simultánea 5/7 (71,4\%).

Del total de pacientes con CI local, 7 (53,8\%) tuvieron infecciones peritoneales relacionadas, en su gran mayoría, a cirugía abdominal, seis los cuales presentaron C. albicans y uno $C$. glabrata. De ellos, cinco pacientes fueron tratados con fluconazol con buena respuesta clínica. Cuatro pacientes tuvieron un cultivo de punta de catéter venoso central positivo, de los cuales dos correspondieron a $C$. albicans, uno a $C$. tropicalis y uno a C. krusei. De ellos, tres pacientes tuvieron hemocultivos simultáneos negativos, dos recibieron tratamiento con fluconazol y uno con anfotericina $\mathrm{B}$ deoxicolato con buena respuesta 


\begin{tabular}{|c|c|c|}
\hline & $\begin{array}{r}\text { Pacien } \\
\mathbf{n} \\
(\mathrm{n}\end{array}$ & $\begin{array}{l}\text { tes con } \mathbf{C l}^{\mathrm{a}} \\
\quad(\%) \\
=53)\end{array}$ \\
\hline \multicolumn{3}{|l|}{ Características demográficas } \\
\hline Edad en años, mediana (rango) & \multicolumn{2}{|c|}{$67(17-92)$} \\
\hline \multicolumn{3}{|l|}{ Género } \\
\hline Mujeres & 31 & $(58,4)$ \\
\hline \multicolumn{3}{|l|}{ Servicio } \\
\hline $\mathrm{UCl} \mathrm{b}^{\mathrm{b}}$ & 40 & $(75,5)$ \\
\hline Intermedio & 13 & $(24,5)$ \\
\hline Estadía hospitalaria días (rango) & \multicolumn{2}{|c|}{$34(8-119)$} \\
\hline $\begin{array}{l}\text { Estadía hospitalaria } \\
\qquad \text { previo a diagnóstico } \mathrm{Cl}\end{array}$ & Estadía hospitalaria & \\
\hline mediana días (rango) & \multicolumn{2}{|c|}{$11 \quad(0-36)$} \\
\hline \multicolumn{3}{|l|}{ Factores de riesgo principales } \\
\hline Antimicrobianos previos & 53 & $(100)$ \\
\hline Uso de CVCc & 41 & $(77,3)$ \\
\hline NPTC $^{d}$ & 20 & $(37,7)$ \\
\hline Cirugía abdominal previa & 13 & $(24,5)$ \\
\hline Uso previo de corticosteroides & 11 & $(20,7)$ \\
\hline \multicolumn{3}{|l|}{ Tipo $^{e}$} \\
\hline Candidemia & 18 & $(33,9)$ \\
\hline $\mathrm{Cl}$ diseminada & 22 & $(41,5)$ \\
\hline $\mathrm{Cl}$ local & 13 & $(24,5)$ \\
\hline \multicolumn{3}{|c|}{$\begin{array}{l}{ }^{a} \mathrm{Cl} \text { : Candidiasis invasora. }{ }^{\mathrm{b}} \mathrm{UCl} \text { : Unidad de cuidados intensivos. 'CVC: } \\
\text { Catéter venoso central. }{ }^{\mathrm{d}} \text { NPTC: Nutrición parenteral total central. } \\
\text { eTipo: se refiere a la forma clínica de candidiasis invasora según las } \\
\text { definiciones establecidas en la sección Pacientes y Métodos. }\end{array}$} \\
\hline
\end{tabular}

\begin{tabular}{|lccccc|}
\hline \multicolumn{6}{|c|}{ Tabla 2. Distribución de especies y susceptibilidad a antifúngicos } \\
en candidemias \\
Especie & n & $(\%)$ & Fluconazol & $\begin{array}{c}\text { Susceptibilidad (\%) } \\
\text { Itraconazol }\end{array}$ & Anfotericina B \\
\hline Candida albicans & 8 & $(44,4)$ & 100 & 100 & 100 \\
Candida no albicans & 10 & $(55,6)$ & 80 & 50 & 100 \\
C. tropicalis & 5 & 100 & 60 & 100 \\
C. glabrata & 2 & 50 & 0 & 100 \\
C. parapsilosis & 1 & 100 & 100 & 100 \\
C. guilliermondii & 1 & 100 & 100 & 100 \\
C. krusei & 1 & 0 & 0 & 100 \\
Total & 18 & $(100)$ & 88,8 & 72,2 & 100 \\
\hline asusceptibilidad realizada por método de difusión-dilución en agar para fluconazol, itraconazol y \\
anfotericina B utilizando E-test (AB Biodisk). b No se realizó susceptibilidad a equinocandinas. \\
\hline
\end{tabular}

clínica. Hubo un paciente con infección pleural por $C$. albicans en el contexto de una perforación de cáncer esofágico y un paciente con cultivo positivo de líquido céfalo-raquídeo a $C$. guilliermondii; sin embargo, fue considerado una contaminación, por lo que no recibió tratamiento, evolucionando satisfactoriamente.

Del total de pacientes ingresados al estudio, 44 (83\%) recibieron tratamiento antifúngico, siendo fluconazol el fármaco más usado (77,3\%). El segundo antifúngico usado fue anfotericina B deoxicolato $(26,4 \%)$ y $3,7 \%$ de los pacientes recibió caspofungina.

La mortalidad hospitalaria global fue de 24,5\%; sin embargo, no fue posible establecer con certeza la mortalidad atribuible a CI, dadas las múltiples variables asociadas. La mortalidad hospitalaria cruda en pacientes con candidemia fue de $16,6 \%$, significativamente más baja que en pacientes con $\mathrm{CI}$ diseminada $(31,8 \%, \mathrm{p}=0,02)$. La mortalidad hospitalaria cruda en pacientes CI local fue de $23,0 \%$. Al comparar por especie de Candida encontrada en candidemias, no hubo diferencias, falleciendo tres pacientes con candidemia por $C$. albicans y tres por Candida no albicans.

\section{Discusión}

La incidencia de CI en los pacientes hospitalizados se ha incrementado sustancialmente durante los últimos años. Estudios recientes han mostrado un aumento de 19 a 24 infecciones por 10.000 egresos hospitalarios/ año entre 1997 y 2003 en E.U.A. ${ }^{19}$. En UCI, algunas instituciones han comunicado un aumento de la incidencia de hasta $487 \%$ en las últimas dos décadas ${ }^{10}$. A pesar de los importantes avances en el tratamiento intensivo y la introducción de nuevas y potentes terapias antifúngicas, la mortalidad de la CI sigue siendo muy elevada y los costos derivados de esta situación muy significativos para las instituciones de salud, estimándose en cifras tan altas como 40.000 dólares por episodio en E.U.A. ${ }^{20}$.

Por otra parte, el tradicional predominio de las infecciones por $C$. albicans ha ido cambiando, observándose un aumento progresivo de infecciones graves causadas por especies de Candida no albicans ${ }^{12}$. Esta situación complica la elección de la terapia antifúngica empírica ya que es difícil predecir la susceptibilidad de las distintas especies de Candida no albicans a los antifúngicos tradicionales $^{15}$. Se estima que en hemocultivos, 15 a $40 \%$ de las C. glabrata y alrededor de $20 \%$ de $C$. tropicalis son resistentes a fluconazol. Candida krusei es intrínsicamente resistente a este antifúngico ${ }^{15,21}$.

El presente estudio confirma estos cambios epidemiológicos en nuestra institución, con un importante aumento de las especies de Candida no albicans aisladas en candidemias $(55,6 \%)$. Sin embargo, a pesar de la 
elevada frecuencia de Candida no albicans y su potencial mayor resistencia a fluconazol comparada con C. albicans, $88,8 \%$ de las cepas aisladas fueron susceptibles a fluconazol, el antifúngico más usado para esta patología en la práctica clínica en nuestro país. Estos datos deben interpretarse teniendo en cuenta que se excluyeron del estudio aquellos pacientes que habían recibido profilaxis o tratamiento previo con antifúngicos, incluyendo azoles, lo que podría reflejar una realidad distinta a aquellos escenarios en los cuales se usa ampliamente profilaxis con fluconazol en la actualidad.

En nuestro país hay poca información respecto a la epidemiología de la candidiasis invasora en UCI. Un estudio multicéntrico ${ }^{22,23}$ en el que participó nuestra institución, evaluó la distribución de especies y susceptibilidad a diversos antifúngicos en aislados de candidiasis invasora (candidemia y cultivos de sitios estériles). Sin embargo, el grupo de pacientes estudiado fue heterogéneo y representa distintas situaciones clínicas, incluyendo pacientes oncológicos, adultos y pediátricos, provenientes de diferentes realidades en los hospitales participantes. En ese estudio se observó que del total de levaduras aisladas en diferentes muestras, $44,6 \%$ correspondió a $C$. albicans y $42,2 \%$ fueron Candida no albicans. El resto de las cepas fueron otros tipos de levaduras ${ }^{22,23}$.

Respecto a la distribución de especies de Candida no albicans en candidemias, Silva y colaboradores ${ }^{22,23}$ encontraron una mayor frecuencia de C. parapsilosis $(20,9 \%)$ seguida por $C$. tropicalis $(14,3 \%)$, lo que difiere de nuestros resultados en los que predominó $C$. tropicalis $(27,7 \%)$ seguida de C. glabrata $(10,5 \%)$. Esta diferencia se podría atribuir a la inclusión de cepas procedentes de neonatos, en los cuales se ha reportado predominancia de $C$. parapsilosis dentro de las cepas de Candida no albicans ${ }^{24,25}$. En relación a susceptibilidad a antifúngicos, todas las cepas de Candida sp aisladas en el estudio mencionado fueron susceptibles a anfotericina B; sin embargo, se observó resistencia a fluconazol en $40 \%$ de las $C$. glabrata y en $17 \%$ de las C. tropicalis estudiadas ${ }^{22,23}$. En nuestro estudio, las cepas de C. tropicalis fueron en 100\% susceptibles a fluconazol y las de $C$. glabrata tuvieron $50 \%$ de resistencia a este antifúngico.

La mortalidad hospitalaria global en nuestra serie $(24,5 \%)$ es menor que la mortalidad reportada recientemente en series más grandes para esta patología, que fluctúan entre 35,2 y 54\% ${ }^{12,26-28}$. Entre otras razones, esto pudiera deberse a las características basales de la población de pacientes estudiados, con una mediana del índice APACHE de 13 puntos que se asocia a una mortalidad de alrededor de $15 \%{ }^{18}$. Sin embargo, llama la atención una mortalidad global significativamente menor en el grupo de pacientes con candidemia comparado a pacientes con $\mathrm{CI}$ diseminada, lo que pudiera deberse al inicio más precoz de antifúngicos en pacientes con hemocultivos positivos. Desafortunadamente, no fue posible explicar este hallazgo con los datos disponibles.

Este estudio tiene algunas limitaciones: si bien nuestros datos reflejan lo que vemos en la práctica clínica diaria en UCI y son concordantes con los cambios epidemiológicos en esta patología, representan la realidad local de pacientes adultos de un sólo hospital y no son necesariamente representativos de otros hospitales o de la situación del resto del país. Por otro lado, a pesar de lo extenso del período de seguimiento y enrolamiento de pacientes, el número total de pacientes es relativamente pequeño, pero permite evaluar y cuantificar la tendencia actual en esta importante situación clínica.

Finalmente, nos parece fundamental continuar el estudio integral de la CI en pacientes críticos no neutropénicos en nuestro país, para así poder evaluar los criterios de diagnóstico clínico y establecer métodos de diagnóstico alternativos como, por ejemplo de biología molecular o determinación de $\beta$-glucanos, que permitan un diagnóstico más sensible y oportuno en esta patología de alta mortalidad y difícil tratamiento.

Agradecimientos. Los autores agradecen sinceramente la valiosa ayuda del personal de los laboratorios de Urgencia y de Microbiología de la Facultad de Medicina, Pontificia Universidad Católica de Chile.

\section{Resumen}

La epidemiología de candidiasis invasora (CI) ha cambiado, lo cual no ha sido suficientemente estudiado en Chile. Objetivo: Describir el perfil epidemiológico y microbiológico de CI en pacientes críticos. Métodos: Estudio observacional prospectivo entre octubre 2001 y agosto 2003, en pacientes críticos adultos con sospecha o confirmación de CI. Resultados: 53 pacientes cumplieron criterios de CI. De ellos, $18(33,9 \%)$ tuvieron candidemia, 22(41,5\%) CI diseminada y $13(24,5 \%) \mathrm{CI}$ local. Entre las candidemias, hubo 8 C. albicans $(44,4 \%)$ y 10 Candida no albicans $(55,6 \%)$, predominando $C$. tropicalis $(27,7 \%)$. Un $88,8 \%$ de las candidemias fueron susceptibles a fluconazol. La mortalidad hospitalaria global fue $24,5 \%$, significativamente menor en pacientes con candidemias vs CI diseminada (16,6 vs 31,8\%, p= 0,02). Conclusiones: Se observó una mayor proporción de Candida no albicans en candidemias de pacientes críticos. Sin embargo, la mayoría de estas cepas fue susceptible a fluconazol. La mortalidad global fue menor en candidemias. 


\section{Referencias}

1.- Wisplinghoff $\mathrm{H}$, Bischoff $\mathrm{T}$, Talent $\mathrm{S} \mathrm{M}$, Seifert H, Wenzel R P, Edmond M B. Nosocomial bloodstream infections in US hospitals: analysis of 24,179 cases from a prospective nationwide surveillance study. Clin Infect Dis 2004; 39: 309-17.

2.- Vincent J L, Anaissie E, Bruining H, Demajo W, el-Ebiary M, Haber J, et al. Epidemiology, diagnosis and treatment of systemic candidal infection in surgical patients under intensive care. Intensive Care Med 1998; 24: 206-16.

3.- Kao A S, Brandt M E, Pruitt W R, Conn L A, Perkins B A, Stephens D S, et al. The epidemiology of candidemia in two United States cities: results of of a population based active surveillance. Clin Infect Dis 1999; 29: $1164-70$

4.- Blumberg H M, Jarvis W R, Soucie J M, Edwards J E, Patterson J E, Pfaller M A, et al. Risk factors for candidal bloodstream infections in surgical intensive care unit patients: The NEMIS prospective multicenter study. Clin Infect Dis 2001; 33: 177-86.

5.- Ostrosky-Zeichner L. New approaches to the risk of Candida in the intensive care unit. Curr Opin Infect Dis 2003; 16: 533-7.

6.- Muñoz P, Burillo A, Bouza E. Criteria used when initiating antifungal therapy against Candida spp in the intensive care unit. Int J Antimicrob Agents 2000; 15: 83-90.

7.- Bodey G, Bueltmann B, Duguid W, Gibbs D, Hanak H, Hotchi M, et al. Fungal infections in cancer patients: an international autopsy survey. Eur J Clin Microbiol Infect Dis 1992; 11: 99 109.

8.- Groll A H, Shah P M, Mentzel C, Schneider M, Just-Nuebling G, Huebner K. Trends in the post mortem epidemiology of invasive fungal infections at a university hospital. J Infect 1996; 33:23-32.

9.- Gudlaugsson O, Gilleespie S, Lee K, Vande Berg J, Hu J, Messer S, et al. Attributable mortality of nosocomial candidemia, revisited.
Clin Infect Dis 2003; 37: 1172-7.

10.- Ostrosky-Zeichner L, Pappas P. Invasive candidiasis in the intensive care unit. Crit Care Med 2006; 34: 857-63.

11.- Garbino J, Kolarova L, Rohner P, Lew D, Pichna P, Pittet D. Secular trends of candidemia over 12 years in adult patients at a tertiary care hospital. Medicine (Baltimore) 2002; 81: 42533.

12.- Horn D, Neofytos D, Anaissie E, Fishman J, Steinbach W, Olyaei A, et al. Epidemiology and outcomes of candidemia in 2010 patients: data from the prospective antifungal therapy alliance registry. Clin Infect Dis 2009; 48: 1695-703.

13.- Gleason T G, May A K, Caparelli D, Farr B M, Sawyer R G. Emerging evidence of selection of fluconazole-tolerant fungi in surgical intensive care units. Arch Surg 1997; 132: 1197-201.

14.- Snydman DR. Shifting patterns in the epidemiology of nosocomial Candida infections. Chest 2003; 123: 500S-503S.

15.- Pappas P, Kauffman C, Andes D, Benjamin D, Calandra T, Edwards J, et al. Clinical practice guidelines for the management of candidiasis: 2009 update by the Infectious Diseases Society of America. Clin Infect Dis 2009; 48: 503-35.

16.- Morrell M, Fraser V J, Kollef M H. Delaying the empiric treatment of Candida bloodstream infection until positive blood culture results are obtained: a potential risk factor for hospital mortality. Antimicrob Agents Chemother 2005; 49(9): 3640-5.

17.- Garey K W, Rege M, Pai M P, Mingo D E, Suda K J, Turpin R S, et al. Time to initiation of fluconazole therapy impacts mortality in patients with candidemia: a multi-institutional study. Clin Infect Dis 2006; 43: 25-31.

18.- Knaus W A, Draper E A, Wagner D P, Zimmerman J E. APACHE II: a severity of disease classification system. Crit Care Med 1985; 13: 818-29.

19.- Pfaller M A, Diekema D J. Epidemiology of invasive candidiasis: a persistent public health problem. Clin Microb Rev 2007; 20: 133-63.

20.- Miller L G, Hajjeh R A, Edwards J E Jr.
Estimating the cost of nosocomial candidemia in the United States. Clin Infect Dis 2001; 32:1110.

21.- Diekema D J, Messer S A, Brueggemann A B, Coffman S L, Doern G V, Herwaldt L A, et al. Epidemiology of candidemia: 3-year results from the emerging infections and the epidemiology of Iowa organims study. J Clin Microbiol 2002; 40: 1298-302.

22.- Silva V, Díaz M C, Febré N y Red de diagnóstico en Micología Médica. Vigilancia de la resistencia de levaduras a antifúngicos. Rev Chil Infect 2002; 19 (Supl 2): S149-56.

23.- Silva V, Díaz M C, Febré N; Chilean Invasive Fungal Infectiones Group. Invasive fungal infections in Chile: a multicenter study of fungal prevalence and susceptibility during a 1-year period. Med Mycol 2004; 42: 333-9.

24.- Pfaller M A, Diekema D J. Role of sentinel surveillance of candidemia: trends in species distribution and antifungal susceptibility. J Clin Microbiol 2002; 40: 3551-7.

25.- Van Asbeck E C, Clemons K V, Stevens D A. Candida parapsilosis: a review of its epidemiology, pathogenesis, clinical aspects, typing and antimicrobial susceptibility. Crit Rev Microbiol 2009; 35: 283-309.

26.- Morgan J, Meltzer M I, Plikaytis B D, Sofair A N, Huie-White S, Wilcox S, et al. Excess mortality, hospital stay, and cost due to candidemia: a case-control study using data from population-based candidemia surveillance. Infect Control Hosp Epidemiol 2005; 26: 540-7.

27.- Zaoutis T E, Argon J, Chu J, Berlin J A, Walsh T I, Feudtner C. The epidemiology and attributable outcomes of candidemia in adults and children hospitalized in the United States: a propensity analysis. Clin Infect Dis 2005; 41 : 1232-9.

28.- Colombo A L, Nucci M, Park B J, Novér S A, Arthington-Skaggs B, da Matta D A, et al. Epidemiology of candidemia in Brazil: a nationwide sentinel surveillance of candidemia in eleven medical centers. J Clin Microbiol 2006; 44: 2816-23. 